\title{
Sweet sequencing
}

Popular crop plants have been bred and selected for desirable taste and color traits. Genomic approaches are increasingly being used to provide insights into the origins, evolution and biology of our favorite foods. Large-scale sequencing efforts have brought agriculture genomics into the big-data era, leading to sweet rewards.

$\mathrm{T}$ his month, we publish two melon genomics studies applying genetics and genomics methods to generate resources for future improvement of these economically important crops. Zhao et al. conduct a large genome re-sequencing study of more than 1,100 melon (Cucumis melo L.) accessions, analyzing global diversity and domestication patterns. They additionally perform genome-wide association studies for different fruit quality traits, identifying significant loci and potential candidate genes for desired traits. Guo et al. present genome re-sequencing of 414 watermelon accessions (Citrullus genus) and provide an improved, high-quality reference genome for this crop. They analyze patterns of speciation and domestication and identify loci that were under selection during these processes. In particular, they characterize the origins and evolution of flesh sweetness, implicating loci regulating raffinose pathways and sugar transport. These large sequencing resources can help lead to sweeter, higher-quality melons. See also the accompanying News \& Views by Martin Mascher.
Sequencing for sweetness is a generalized strategy. Just in this past year, the pineapple genome as well as the strawberry genome were published in Nature Genetics. These food plants, along with melons, are economically important and have enormous markets; they are eaten and enjoyed throughout the world. Taste, then, can be just as important a trait as yield or shelf life.

Agricultural genomics research has the goal of improving the quality and yield of the crops that we rely on as sources of nutrients and calories. Importantly, the United Nations Sustainable Development Goals, aiming to address global challenges surrounding health and equality, include 'zero hunger' as a critical objective. Although genomics methods can be used to obtain sweeter melons, crisper apples or redder tomatoes, they are also being used to produce heartier staple crops, such as drought-tolerant rice or disease-resistant wheat. Food provides both pleasure and sustenance, and crop genetics can facilitate both qualities.

Therefore, it seems appropriate to highlight the launch of a new journal within the Nature family, Nature Food, which is now open for submissions. This comprehensive journal will publish studies related to all aspects of food, from production to consumption, and as such, it will provide a dedicated perspective to food-related issues that will help put agricultural genetics research into a broader context. Look for the first publications in January 2020.

For even more delicious developments, later this month, Nature Genetics will attend our Nature Conference 'Agricultural Genomics: Big Data for Better Agriculture' in Shenzhen, China. We are eager to learn about the current genomic innovations being implemented for different crops across diverse environments, and to see how plant geneticists are harnessing big data to help improve crops and feed the world. We are sure that the fruits of their labor will be sweet.

Published online: 1 November 2019 https://doi.org/10.1038/s41588-019-0533-5 